\title{
Global Stability for a Discrete Space-Time Lotka-Volterra System with Feedback Control
}

\author{
Li Xu $\mathbb{D D}^{1,2}$ and Ruiwen Han ${ }^{1}$ \\ ${ }^{1}$ School of Science, Tianjin University of Commerce, Tianjin 300134, China \\ ${ }^{2}$ School of Mathematics, Tianjin University, Tianjin 300072, China \\ Correspondence should be addressed to Li Xu; beifang_xl@163.com
}

Received 26 May 2020; Revised 11 July 2020; Accepted 27 July 2020; Published 19 August 2020

Academic Editor: Juan Carlos Cortés

Copyright (c) $2020 \mathrm{Li} \mathrm{Xu}$ and Ruiwen Han. This is an open access article distributed under the Creative Commons Attribution License, which permits unrestricted use, distribution, and reproduction in any medium, provided the original work is properly cited.

\begin{abstract}
In this paper, a discrete space-time Lotka-Volterra model with the periodic boundary conditions and feedback control is proposed. By means of a discrete version of comparison theorem, the boundedness of the nonnegative solution of the system is proved. By the combination of the Volterra-type and quadratic Lyapunov functions, the global asymptomatic stability of the unique positive equilibrium is investigated. Finally, numerical simulations are presented to verify the effectiveness of the main results.
\end{abstract}

\section{Introduction}

It is well known that the ecosystem in the real world is often distributed by unpredictable forces or interference factors, such as natural disturbances (floods, fires, disease outbreaks, and droughts), human-caused interference factors (oil spills), and slowly changing long-term stresses (nutrient enrichment), which may result into changes in the biological parameters such as survival rates [1-3]. The presence of the unpredictable forces or interference factors in an ecological system raises the following essential and basic question from the practical interest in ecology: "Can the ecosystem withstand those unpredictable forces which persist for a finite period of time?" The question has motivated the development of some control mechanisms for managing populations to ensure that the interacting species can coexist, such as impulsive control, optimal vibration control, intermittent control, and feedback control. [4-6]. As a basic mechanism by which one can recover stability and move the trajectory towards the desired orbit, the introduction of a feedback control variable is one method that can achieve the objective.

For population dynamical systems with feedback controls, an important and interesting subject is to study the effects of feedback controls to the persistence, permanence, and extinction of species, the stability, and dynamical complexity of systems [7]. There are lots of important and interesting results on stability research for continuous time population dynamical models [8-16]. A necessary condition for sustained concentration oscillations resulting from small perturbations of the steady state is derived from a closure rule using a variation of the direct Lyapunov method on a biochemical feedback system of the Yates-Pardee type [8]. The authors study the dynamical behavior of a continuous reaction-diffusion waterborne pathogen model, such as the existence of positive solutions and its boundedness, the existence of equilibria, local stability, uniform persistence, and global stability [9]. The output feedback stabilization of stochastic feedforward systems with unknown control coefficients and unknown output function using the timevarying technique and backstepping method is achieved [16].

The discrete-time models governed by difference equation are more realistic than the continuous ones when the populations have nonoverlapping generations or the population statistics are compiled from given time intervals and not continuously. Moreover, discrete-time models can also provide efficient computational models of continuous 
models for numerical simulations. Therefore, it is reasonable to study discrete-time models governed by difference equations, and there has been some work done on the study of the persistence, permanence, and global stability for various discrete-time nonlinear population systems with feedback when the effect of spatial factors is not considered $[5,7,17-19]$. A weak sufficient condition for the permanence of a nonautonomous discrete single-species system with delays and feedback control is given in the article [7]. A twospecies competitive system with feedback controls is considered, in which the global attractivity of a positive periodic solution is obtained, and the existence and uniqueness of the uniformly asymptotically stable almost periodic solution are shown $[17,18]$. In reference [19], some sufficient conditions on the permanence and the global stability of the system of a $n$-species Lotka-Volterra discrete system with delays and feedback control by constructing the suitable discrete type Lyapunov functionals are obtained.

It is a fact that spatial heterogeneity and dispersal play an important role in the dynamics of populations, which has been the subject of much research, both theoretical and experimental, such as the role of dispersal in the maintenance of patchiness or spatial population variation. If the spatial factors are added, more dynamics will occur. The diffusion-driven instability may emerge if the steadystate solution is stable to small spatial perturbations in absence of diffusion, but unstable when diffusion is present [20]. If the diffusion-driven instability should be avoided in some situations, and one may wish recovery stability towards the desired orbit, but the system parameters are not easy to adjust, then some other ways should be adopted to achieve the stabilization aim [21]. There also may exist a situation where the equilibrium of the dynamical model is not the desirable one (or affordable) and a smaller value of the equilibrium is required; then, altering the model structure so as to make the population stabilize at a lower value is necessary [22]. Feedback control will be an effective one and can alter the positions of positive equilibrium or obtain its stability. To the best of our knowledge, there is few work that has been devoted to global properties of the discrete space-time models with feedback control. The robustly asymptotic stability and disturbance attenuation level of the filtering error system for a two-dimensional Roesser models with polytopic uncertainties are discussed [23]. A two-dimensional Fornasini-Marchesini local state-space system is also considered in the article [24]. However, the diffusion terms (discrete Laplace operator) are not directly introduced into the model. There is some work on global stability of discrete diffusion systems $[25,26]$, in which the positivity, boundedness, and global stability of the equilibria are established, and the discretized models are derived from the corresponding continuous model by nonstandard finite difference, but the Laplace operator has been dealt with. It is a fact that diffusion will produce much richer dynamical behaviors and complexity; how to analyze stability of the discrete diffusion system with feedback control by means of suitable Lyapunov functions is an important problem to solve.
Motivated by above discussions, the main purpose of this paper is to study the global asymptomatic stability of an one-dimensional spatially discrete reaction diffusion Lotka-Volterra model with the periodic boundary conditions and feedback control. So the organization of this paper is as follows. In the Section 2, we formulate the discrete space-time Lotka-Volterra model with feedback control and present some assumptions and preparations which will be essential to our main proofs, and the nonnegativity and boundedness of the solution of the system are proved by means of comparison theorem. Then, global asymptotic stability of the unique positive equilibrium is proved by constructing a combination of the nonnegative Volterra-type and quadratic Lyapunov functions in Section 3. In Section 4, numerical simulations are presented to illustrate the feasibility of our main results. In the last section, brief discussions and conclusions are given.

\section{Model and Preliminaries}

It is well known that a Lotka-Volterra system can be described in the form of

$$
\begin{aligned}
& x^{\prime}(t)=x(t)\left(r_{1}-a_{11} x(t)-a_{12} y(t)\right), \\
& y^{\prime}(t)=y(t)\left(r_{2}+a_{21} x(t)-a_{22} y(t)\right),
\end{aligned}
$$

which is called the predator-prey model. $x(t)$ is the density of prey species, $y(t)$ is the density of predator species, the coefficients $a_{11}$ and $a_{22}$ represent the intraspecific interactions, $a_{12}$ and $a_{21}$ represent the interspecific interactions, and $r_{1}$ and $r_{2}$ are the intrinsic growth rates of the respective species.

A corresponding discrete model for the system (1) can be derived from [27]:

$$
\left\{\begin{array}{l}
x_{n+1}=x_{n} \exp \left(r_{1}-a_{11} x_{n}-a_{12} y_{n}\right), \\
y_{n+1}=y_{n} \exp \left(r_{2}+a_{21} x_{n}-a_{22} y_{n}\right),
\end{array}\right.
$$

where $a_{i j}(i, j=1,2)>0$. Let $X_{n}=a_{11} x_{n}$ and $Y_{n}=a_{22} y_{n}$; we have

$$
\begin{aligned}
& \left\{\begin{array}{l}
X_{n+1}=X_{n} \exp \left(r_{1}-X_{n}-\frac{a_{12}}{a_{22}} Y_{n}\right), \\
Y_{n+1}=Y_{n} \exp \left(r_{2}+\frac{a_{21}}{a_{11}} X_{n}-Y_{n}\right),
\end{array}\right. \\
& \text { or }\left\{\begin{array}{l}
x_{n+1}=x_{n} \exp \left(r_{1}-x_{n}-a_{12} y_{n}\right), \\
y_{n+1}=y_{n} \exp \left(r_{2}+a_{21} x_{n}-y_{n}\right) .
\end{array}\right.
\end{aligned}
$$

It is believed that the diffusion of individuals can play an important role in determining collective behavior of the population. Space factors can be taken into account in all fundamental aspects of ecological organization, and we can get a one-dimensional discrete reaction-diffusion model as follows: 


$$
\left\{\begin{array}{l}
x_{i}^{n+1}=x_{i}^{n} \exp \left(r_{1}-x_{i}^{n}-a_{12} y_{i}^{n}\right)+D_{1} \nabla^{2} x_{i}^{n}, \\
y_{i}^{n+1}=y_{i}^{n} \exp \left(r_{2}+a_{21} x_{i}^{n}-y_{i}^{n}\right)+D_{2} \nabla^{2} y_{i}^{n},
\end{array}\right.
$$

where $i \in\{1,2, \ldots, m\}=[1, m]$ and $m, n \in Z^{+}$are positive integers and $D_{1}$ and $D_{2}$ are diffusion parameters.

$$
\begin{aligned}
& \nabla^{2} x_{i}^{n}=x_{i+1}^{n}-2 x_{i}^{n}+x_{i-1}^{n}, \\
& \nabla^{2} y_{i}^{n}=y_{i+1}^{n}-2 y_{i}^{n}+y_{i-1}^{n} .
\end{aligned}
$$

This also indicates the coupling or diffusion from the units or individuals to the left and the right, respectively. The following periodic boundary conditions are considered:

$$
\left\{\begin{array}{l}
x_{0}^{n}=x_{m}^{n}, x_{1}^{n}=x_{m+1}^{n} \\
y_{0}^{n}=y_{m}^{n}, y_{1}^{n}=y_{m+1}^{n}
\end{array}\right.
$$

Systems (4)-(6) can exhibit rich dynamic behaviors, and diffusion-driven instability may emerge [28]. As discussed in the introduction part, unpredictable forces or interference factors can be introduced into the forms of feedback control variables, which can contribute significantly to the biological systems by affecting their dynamics and stability. Moreover, structurally modifying existing systems by incorporating variables for defining feedback controls is appropriate for considering the unpredictable forces or interference factors in an ecosystem. So, in the present study, we consider the following one-dimensional discrete space-time Lotka-Volterra model with periodic boundary conditions and feedback control:

$$
\left\{\begin{array}{l}
x_{i}^{n+1}=x_{i}^{n} \exp \left(r_{1}-x_{i}^{n}-a_{12} y_{i}^{n}-d_{1} u_{1 i}^{n}\right)+D_{1} \nabla^{2} x_{i}^{n}, \\
y_{i}^{n+1}=y_{i}^{n} \exp \left(r_{2}+a_{21} x_{i}^{n}-y_{i}^{n}-d_{2} u_{2 i}^{n}\right)+D_{2} \nabla^{2} y_{i}^{n}, \\
u_{1 i}^{n+1}=\left(1-\eta_{1}\right) u_{1 i}^{n}+e_{1} x_{i}^{n}, \\
u_{2 i}^{n+1}=\left(1-\eta_{2}\right) u_{2 i}^{n}+e_{2} y_{i}^{n},
\end{array}\right.
$$

with the periodic boundary conditions

$$
\left\{\begin{array}{l}
x_{0}^{n}=x_{m}^{n}, x_{1}^{n}=x_{m+1}^{n}, \\
y_{0}^{n}=y_{m}^{n}, y_{1}^{n}=y_{m+1}^{n},
\end{array}\right.
$$

where $i \in\{1,2, \ldots, m\}=[1, m]$ and $m, n \in Z^{+}$is positive integer, $r_{1}, r_{2}, a_{12}, a_{21}, \eta_{1}, \eta_{2}, e_{1}, e_{2}$ are positive constants, and $D_{1}$ and $D_{2}$ are diffusion parameters.

$$
\begin{aligned}
& \nabla^{2} x_{i}^{n}=x_{i+1}^{n}-2 x_{i}^{n}+x_{i-1}^{n}, \\
& \nabla^{2} y_{i}^{n}=y_{i+1}^{n}-2 y_{i}^{n}+y_{i-1}^{n} .
\end{aligned}
$$

To the best of our knowledge, no work on global asymptomatic stability of the positive equilibrium of systems (7) and (8) has been done yet.

By simple computation, systems (7) and (8) have a positive equilibrium:

$$
E^{*}=\left(x^{*}, y^{*}, u_{1}^{*}, u_{2}^{*}\right),
$$

where

$$
\begin{aligned}
x^{*} & =\frac{\eta_{1} r_{1}\left(\eta_{2}+e_{2} d_{2}\right)-\eta_{1} \eta_{2} a_{12} r_{2}}{\left(\eta_{2}+e_{2} d_{2}\right)\left(\eta_{1}+e_{1} d_{1}\right)+a_{12} a_{21} \eta_{1} \eta_{2}}, \\
y^{*} & =\frac{\eta_{2} r_{2}\left(\eta_{1}+e_{1} d_{1}\right)+\eta_{1} \eta_{2} a_{21} r_{1}}{\left(\eta_{2}+e_{2} d_{2}\right)\left(\eta_{1}+e_{1} d_{21}\right)+a_{12} a_{21} \eta_{1} \eta_{2}}, \\
u_{1}^{*} & =\frac{e_{1}}{\eta_{1}} x^{*}, \\
u_{2}^{*} & =\frac{e_{2}}{\eta_{2}} y^{*} .
\end{aligned}
$$

If $r_{1}\left(\eta_{2}+e_{2} d_{2}\right)>\eta_{2} a_{12} r_{2}$, the equilibrium is positive.

To discuss the global asymptomatic stability of the unique positive equilibrium, the following assumptions and preparations are essential.

From the view point of biology, we only need to discuss the positive solution of system (7). So, it is assumed that the initial conditions of (8) are of the form

$$
x_{i}^{0}>0, u_{i}^{0}>0, \quad i=1,2, \ldots, m .
$$

For our purpose, we first introduce the following lemma which can be obtained easily by comparison theorem of difference equation.

Lemma 1 (see [29]). Let $x(n)$ be a nonnegative solution of inequality

$$
x(n+1) \leq x(n) \exp \{\alpha-\beta x(n)\}, \quad n \in Z,
$$

with $x(0)>0$ and $\alpha, \beta>0$; then,

$$
\lim _{n \longrightarrow \infty} \sup x(n) \leq \frac{\alpha}{\beta} \text {. }
$$

Lemma 2 (see [30]). Any solution $x(n)$ of system

$$
x(n+1)=x(n)(1-\gamma)+\omega(n), \quad n \in Z
$$

with $x(0)>0$ satisfies

$$
\lim _{n \longrightarrow \infty} \sup x(n) \leq \frac{\operatorname{up} n \in Z \omega(n)}{\gamma},
$$

where $\omega(n)$ is a nonnegative bounded sequence of real numbers and $0<\gamma<1$.

Applying the above lemmas, we can obtain the following result.

Theorem 1. The solution of (7) with initial condition (8) is defined and remains nonnegative and bounded if $e^{r_{j}}-2 D_{j} \geq 0$ and $\eta_{j}<1, j=1,2$ hold.

Proof. From the first equation of system (7), we get

$$
\begin{aligned}
x_{i}^{n+1}= & x_{i}^{n} \exp \left(r_{1}-x_{i}^{n}-a_{12} y_{i}^{n}-d_{1} u_{1 i}^{n}\right)+D_{1} \nabla^{2} x_{i}^{n} \\
= & x_{i}^{n}\left(\exp \left(r_{1}-x_{i}^{n}-a_{12} y_{i}^{n}-d_{1} u_{1 i}^{n}\right)-2 D_{1}\right) \\
& +D_{1}\left(x_{i+1}^{n}+x_{i-1}^{n}\right),
\end{aligned}
$$


from which it is true that $x_{i}^{n} \geq 0$ holds for all $n$ with $x_{i}^{0}>0, u_{i}^{0}>0, i=1,2, \ldots, m$ if $e^{r_{1}}-2 D_{1} \geq 0$ and appropriate parameters $a_{12}, d_{1}$ are selected.

Similarly, from the second equation of system (7), we get that $y_{i}^{n} \geq 0$ holds for all $n$ with $x_{i}^{0}>0, u_{i}^{0}>0, i=1,2, \ldots, m$, if $e^{r_{2}}-2 D_{2} \geq 0$ and appropriate parameters $a_{21}, d_{2}$ are selected.

If $\eta_{j} \leq 1, j=1,2, u_{j i}^{n} \geq 0$ can also hold by means of the third and fourth equations of system (7).

Next, we will show the boundedness of the solutions.

$$
\begin{aligned}
\sum_{i=1}^{m} x_{i}^{n+1} & =\sum_{i=1}^{m}\left(x_{i}^{n} \exp \left(r_{1}-x_{i}^{n}-a_{12} y_{i}^{n}-d_{1} u_{1 i}^{n}\right)+D_{1} \nabla^{2} x_{i}^{n}\right) \\
& =\sum_{i=1}^{m} x_{i}^{n} \exp \left(r_{1}-x_{i}^{n}-a_{12} y_{i}^{n}-d_{1} u_{1 i}^{n}\right) \\
& \leq \sum_{i=1}^{m} x_{i}^{n} \exp \left(r_{1}-x_{i}^{n}\right) .
\end{aligned}
$$

From Lemma 1, we can obtain

$$
\lim _{n \longrightarrow \infty} \sup \sum_{i=1}^{m} x_{i}^{n} \leq \sum_{i=1}^{m} r_{1}=m r_{1} .
$$

Similarly, we can also obtain

$$
\begin{aligned}
\sum_{i=1}^{m} y_{i}^{n+1} & =\sum_{i=1}^{m}\left(y_{i}^{n} \exp \left(r_{2}+a_{21} x_{i}^{n}-y_{i}^{n}-d_{2} u_{2 i}^{n}\right)+D_{2} \nabla^{2} y_{i}^{n}\right) \\
& =\sum_{i=1}^{m} y_{i}^{n} \exp \left(r 2+a_{21} x_{i}^{n}-y_{i}^{n}-d_{2} u_{2 i}^{n}\right) \\
& \leq \sum_{i=1}^{m} y_{i}^{n} \exp \left(r_{2}+a_{21} M_{x}-y_{i}^{n}\right),
\end{aligned}
$$

where $M_{x}=\sup _{n \in Z} x_{i}^{n}$. Then,

$$
\lim _{n \longrightarrow \infty} \sup \sum_{i=1}^{m} y_{i}^{n} \leq \sum_{i=1}^{m}\left(r_{2}+a_{21} M_{x}\right)=m\left(r_{2}+a_{21} M_{x}\right) .
$$

From Lemma 2, by means of (20) and (21) and $\eta_{1}, \eta_{2} \leq 1$, we can obtain

$$
\begin{aligned}
& \lim _{n \longrightarrow \infty} \sup u_{1 i}^{n} \leq \frac{e_{1} M_{x}}{\eta_{1}}, \\
& \lim _{n \longrightarrow \infty} \sup u_{2 i}^{n} \leq \frac{e_{2} M_{y}}{\eta_{2}},
\end{aligned}
$$

where $M_{y}=\sup _{n \in Z} y_{i}^{n}$. The proof is finished.

\section{Global Stability}

In this section, we devote ourselves to studying the global asymptotic stability of the unique positive equilibrium $E^{*}$. By using global Lyapunov function, we derive the sufficient conditions under which the positive equilibrium is globally asymptotically stable.

Denote

$$
\begin{aligned}
& H(1): e^{r_{j}}-2 D_{j} \geq 0, \quad j=1,2, \\
& H(2): \frac{d_{j} e_{j}}{2\left(1-\eta_{j}\right)} \leq 1, \quad \eta_{j} \leq 1, j=1,2 .
\end{aligned}
$$

Assume $\left\{x_{i}^{n}\right\}_{i \in[1, m]}^{n \in Z^{+}},\left\{y_{i}^{n}\right\}_{i \in[1, m]}^{n \in Z^{+}}$are positive solutions of systems (7) and (8); we can establish the following result.

Theorem 2. Assume $H(1)$ and $H(2)$ hold; the positive equilibrium $E^{*}$ of systems (7) and (8) is globally asymptotically stable.

Proof. Let

$$
V_{1}^{n}=\sum_{i=1}^{m}\left(x_{i}^{n}-x^{*}-x^{*} \ln \frac{x_{i}^{n}}{x^{*}}\right) .
$$

Then, we can obtain

$$
\begin{aligned}
& \Delta V_{1}^{n}=V_{1}^{n+1}-V_{1}^{n} \\
& =\sum_{i=1}^{m}\left(x_{i}^{n+1}-x_{i}^{n}-x^{*} \ln \frac{x_{i}^{n+1}}{x_{i}^{n}}\right) \\
& =\sum_{i=1}^{m}\left(x_{i}^{n+1}-x_{i}^{n}-x^{*} \frac{x_{i}^{n+1}-x_{i}^{n}}{x_{i}^{n}}\right)+o(1) \\
& =\sum_{i=1}^{m}\left(x_{i}^{n+1}-x_{i}^{n}\right)\left(1-\frac{x^{*}}{x_{i}^{n}}\right)+o(1) \\
& =\sum_{i=1}^{m}\left(1-\frac{x^{*}}{x_{i}^{n}}\right)\left(x_{i}^{n} \exp \left(r_{1}-x_{i}^{n}-a_{12} y_{i}^{n}-d_{1} u_{1 i}^{n}\right)\right. \\
& \left.+D_{1} \nabla^{2} x_{i}^{n}-x_{i}^{n}\right)+o(1) \\
& =\sum_{i=1}^{m}\left(1-\frac{x^{*}}{x_{i}^{n}}\right)\left(x _ { i } ^ { n } \left(1-\left(x_{i}^{n}-x^{*}\right)-a_{12}\left(y_{i}^{n}-y^{*}\right)\right.\right. \\
& \left.\left.-d_{1}\left(u_{1 i}^{n}-u_{1}^{*}\right)\right)+D_{1} \nabla^{2} x_{i}^{n}-x_{i}^{n}\right) \\
& -D_{1} \sum_{i=1}^{m} x^{*}\left(\frac{x_{i+1}^{n}}{x_{i}^{n}}+\frac{x_{i-1}^{n}}{x_{i}^{n}}-2\right)+o(1)+o\left(\rho_{1}\right) \\
& =-\sum_{i=1}^{m}\left(x_{i}-x_{i}^{*}\right)^{2}-a_{12} \sum_{i=1}^{m}\left(x_{i}^{n}-x^{*}\right)\left(y_{i}^{n}-y^{*}\right) \\
& -d_{1} \sum_{i=1}^{m}\left(x_{i}^{n}-x^{*}\right)\left(u_{1 i}^{n}-u_{1}^{*}\right)-D_{1} x^{*} \sum_{i=1}^{m-1} \\
& \cdot\left(\sqrt{\frac{x_{i+1}^{n}}{x_{i}^{n}}}-\sqrt{\frac{x_{i-1}^{n}}{x_{i}^{n}}}\right)^{2}-D_{1} x^{*}\left(\sqrt{\frac{x_{m}^{n}}{x_{1}^{n}}}-\sqrt{\frac{x_{1}^{n}}{x_{m}^{n}}}\right)^{2} \\
& +o(1)+o\left(\rho_{1}\right) \text {, }
\end{aligned}
$$

where $\rho_{1}=\sqrt{\left(x_{i}^{n}-x^{*}\right)^{2}+\left(y_{i}^{n}-y^{*}\right)^{2}+\left(u_{1 i}^{n}-u_{1}^{*}\right)^{2}}$. 
Let

Then, we can obtain

$V_{2}^{n}=\frac{a_{12}}{a_{21}} \sum_{i=1}^{m}\left(y_{i}^{n}-y^{*}-y^{*} \ln \frac{y_{i}^{n}}{y^{*}}\right)$.

$$
\begin{aligned}
& \Delta V_{2}^{n}=V_{2}^{n+1}-V_{2}^{n} \\
& =\frac{a_{12}}{a_{21}} \sum_{i=1}^{m}\left(y_{i}^{n+1}-y_{i}^{n}-y^{*} \ln \frac{y_{i}^{n+1}}{y_{i}^{n}}\right) \\
& =\frac{a_{12}}{a_{21}} \sum_{i=1}^{m}\left(y_{i}^{n+1}-y_{i}^{n}-y^{*} \frac{y_{i}^{n+1}-y_{i}^{n}}{y_{i}^{n}}\right)+o(1) \\
& =\frac{a_{12}}{a_{21}} \sum_{i=1}^{m}\left(y_{i}^{n+1}-y_{i}^{n}\right)\left(1-\frac{y^{*}}{y_{i}^{n}}\right)+o(1) \\
& =\frac{a_{12}}{a_{21}} \sum_{i=1}^{m}\left(1-\frac{y^{*}}{y_{i}^{n}}\right)\left(y_{i}^{n} \exp \left(r_{2}+a_{21} x_{i}^{n}-y_{i}^{n}-d_{2} u_{2 i}^{n}\right)+D_{2} \nabla^{2} y_{i}^{n}-y_{i}^{n}\right)+o(1) \\
& =\frac{a_{12}}{a_{21}} \sum_{i=1}^{m}\left(1-\frac{y^{*}}{y_{i}^{n}}\right)\left(y_{i}^{n}\left(1-\left(y_{i}^{n}-y^{*}\right)+a_{21}\left(x_{i}^{n}-x^{*}\right)-d_{2}\left(u_{2 i}^{n}-u_{2}^{*}\right)\right)-y_{i}^{n}+D_{2} \nabla^{2} y_{i}^{n}\right)+o(1)+o\left(\rho_{2}\right) \\
& =\frac{a_{12}}{a_{21}} \sum_{i=1}^{m}\left(y_{i}^{n}-y^{*}\right)\left(-\left(y_{i}^{n}-y^{*}\right)+a_{21}\left(x_{i}^{n}-x^{*}\right)-d_{2}\left(u_{2 i}^{n}-u_{2}^{*}\right)\right)-D_{2} \frac{a_{12}}{a_{21}} \sum_{i=1}^{m} y^{*}\left(\frac{y_{i+1}^{n}}{y_{i}^{n}}+\frac{y_{i-1}^{n}}{y_{i}^{n}}-2\right)+o(1)+o\left(\rho_{2}\right) \\
& =-\frac{a_{12}}{a_{21}} \sum_{i=1}^{m}\left(y_{i}-y_{i}^{*}\right)^{2}+\sum_{i=1}^{m} a_{12}\left(x_{i}^{n}-x^{*}\right)\left(y_{i}^{n}-y^{*}\right)-\frac{a_{12} d_{2}}{a_{21}} \sum_{i=1}^{m}\left(y_{i}^{n}-y^{*}\right)\left(u_{2 i}^{n}-u_{2}^{*}\right)-D_{2} \frac{a_{12}}{a_{21}} \sum_{i=1}^{m-1} \\
& \cdot\left(\sqrt{\frac{y_{i+1}^{n}}{y_{i}^{n}}}-\sqrt{\frac{y_{i-1}^{n}}{y_{i}^{n}}}\right)^{2}-D_{2} \frac{a_{12}}{a_{21}}\left(\sqrt{\frac{y_{m}^{n}}{y_{1}^{n}}}-\sqrt{\frac{y_{1}^{n}}{y_{m}^{n}}}\right)^{2}+o(1)+o\left(\rho_{2}\right)
\end{aligned}
$$

$$
\begin{aligned}
\Delta V_{2}^{n}= & V_{2}^{n+1}-V_{2}^{n} \\
= & \frac{a_{12}}{a_{21}} \sum_{i=1}^{m}\left(y_{i}^{n+1}-y_{i}^{n}-y^{*} \ln \frac{y_{i}^{n+1}}{y_{i}^{n}}\right) \\
= & \frac{a_{12}}{a_{21}} \sum_{i=1}^{m}\left(y_{i}^{n+1}-y_{i}^{n}-y^{*} \frac{y_{i}^{n+1}-y_{i}^{n}}{y_{i}^{n}}\right)+o(1) \\
= & \frac{a_{12}}{a_{21}} \sum_{i=1}^{m}\left(y_{i}^{n+1}-y_{i}^{n}\right)\left(1-\frac{y^{*}}{y_{i}^{n}}\right)+o(1) \\
= & \frac{a_{12}}{a_{21}} \sum_{i=1}^{m}\left(1-\frac{y^{*}}{y_{i}^{n}}\right)\left(y_{i}^{n} \exp \left(r_{2}+a_{21} x_{i}^{n}-y_{i}^{n}-d_{2} u_{2 i}^{n}\right)+D_{2} \nabla^{2} y_{i}^{n}-y_{i}^{n}\right)+o(1) \\
= & \frac{a_{12}}{a_{21}} \sum_{i=1}^{m}\left(1-\frac{y^{*}}{y_{i}^{n}}\right)\left(y_{i}^{n}\left(1-\left(y_{i}^{n}-y^{*}\right)+a_{21}\left(x_{i}^{n}-x^{*}\right)-d_{2}\left(u_{2 i}^{n}-u_{2}^{*}\right)\right)-y_{i}^{n}+D_{2} \nabla^{2} y_{i}^{n}\right)+o(1)+o\left(\rho_{2}\right) \\
= & \frac{a_{12}}{a_{21}} \sum_{i=1}^{m}\left(y_{i}^{n}-y^{*}\right)\left(-\left(y_{i}^{n}-y^{*}\right)+a_{21}\left(x_{i}^{n}-x^{*}\right)-d_{2}\left(u_{2 i}^{n}-u_{2}^{*}\right)\right)-D_{2} \frac{a_{12}}{a_{21}} \sum_{i=1}^{m} y^{*}\left(\frac{y_{i+1}^{n}}{y_{i}^{n}}+\frac{\left.y_{i-1}^{n}-2\right)+o(1)+o\left(\rho_{2}\right)}{y_{i}^{n}}\right. \\
= & -\frac{a_{12}}{a_{21}} \sum_{i=1}^{m}\left(y_{i}-y_{i}^{*}\right)^{2}+a_{12} \sum_{i=1}^{m}\left(x_{i}^{n}-x^{*}\right)\left(y_{i}^{n}-y^{*}\right)-\frac{a_{12} d_{2}}{a_{21}} \sum_{i=1}^{m}\left(y_{i}^{n}-y^{*}\right)\left(u_{2 i}^{n}-u_{2}^{*}\right)-D_{2} \frac{a_{12}}{a_{21}} \sum_{i=1}^{m-1}\left(\sqrt{\frac{y_{i+1}^{n}}{y_{i}^{n}}}-\sqrt{\frac{y_{i-1}^{n}}{y_{i}^{n}}}\right)^{2} \\
& -D_{2} \frac{a_{12}}{a_{21}}\left(\sqrt{\left.\frac{y_{m}^{n}}{y_{1}^{n}}-\sqrt{\frac{y_{1}^{n}}{y_{m}^{n}}}\right)^{2}+o(1)+o\left(\rho_{2}\right),}\right.
\end{aligned}
$$


where $\rho_{2}=\sqrt{\left(x_{i}^{n}-x^{*}\right)^{2}+\left(y_{i}^{n}-y^{*}\right)^{2}+\left(u_{2 i}^{n}-u_{2}^{*}\right)^{2}}$.

Let

$$
V_{3}^{n}=\frac{d_{1}}{2\left(1-\eta_{1}\right) e_{1}}\left(u_{1 i}^{n}-u_{1}^{*}\right)^{2} .
$$

Then, we can obtain

$$
\begin{aligned}
\Delta V_{3}^{n}= & V_{3}^{n+1}-V_{3}^{n} \\
= & \frac{d_{1}}{2\left(1-\eta_{1}\right) e_{1}} \sum_{i=1}^{m}\left(u_{1 i}^{n+1}-u_{1 i}^{n}\right)\left(u_{1 i}^{n+1}+u_{1 i}^{n}-2 u_{1}^{*}\right) \\
= & \frac{d_{1}}{2\left(1-\eta_{1}\right) e_{1}} \sum_{i=1}^{m}\left(-\eta_{1} u_{1 i}^{n}+e_{1} x_{i}^{n}\right)\left(\left(2-\eta_{1}\right) u_{1 i}^{n}\right. \\
& \left.+e_{1} x_{i}^{n}-2 u_{1}^{*}\right) \\
= & \frac{d_{1}}{2\left(1-\eta_{1}\right) e_{1}} \sum_{i=1}^{m}\left(-\eta_{1}\left(u_{1 i}^{n}-u_{1}^{*}\right)+e_{1}\left(x_{i}^{n}-x^{*}\right)\right) \\
& \cdot\left(\left(2-\eta_{1}\right)\left(u_{1 i}^{n}-u_{1}^{*}\right)+e_{1}\left(x_{i}^{n}-x^{*}\right)\right) \\
= & -\frac{d_{1} \eta_{1}\left(2-\eta_{1}\right)}{2\left(1-\eta_{1}\right) e_{1}} \sum_{i=1}^{m}\left(u_{1 i}^{n}-u_{1}^{*}\right)^{2} \\
& +\frac{d_{1} e_{1}}{2\left(1-\eta_{1}\right)} \sum_{i=1}^{m}\left(x_{i}^{n}-x^{*}\right)^{2}+d_{1}\left(x_{i}^{n}-x^{*}\right)\left(u_{1 i}^{n}-u_{1}^{*}\right) .
\end{aligned}
$$

Let

$$
V_{4}^{n}=\frac{d_{2} a_{12}}{2\left(1-\eta_{2}\right) e_{2} a_{21}}\left(u_{2 i}^{n}-u_{2}^{*}\right)^{2} .
$$

Then, we can obtain

$$
\begin{aligned}
\Delta V_{4}^{n}= & V_{4}^{n+1}-V_{4}^{n} \\
= & \frac{d_{2} a_{12}}{2\left(1-\eta_{2}\right) e_{2} a_{21}} \sum_{i=1}^{m}\left(u_{2 i}^{n+1}-u_{2 i}^{n}\right)\left(u_{2 i}^{n+1}+u_{2 i}^{n}-2 u_{2}^{*}\right) \\
= & \frac{d_{2} a_{12}}{2\left(1-\eta_{2}\right) e_{2} a_{21}} \sum_{i=1}^{m}\left(-\eta_{2} u_{2 i}^{n}+e_{2} y_{i}^{n}\right)\left(\left(-\eta_{2}\right) u_{2 i}^{n}\right. \\
& \left.+e_{2} y_{i}^{n}-2 u_{2}^{*}\right) \\
= & \frac{d_{2} a_{12}}{2\left(1-\eta_{2}\right) e_{2} a_{21}} \sum_{i=1}^{m}\left(-\eta_{2}\left(u_{2 i}^{n}-u_{2}^{*}\right)\right. \\
& \left.+e_{2}\left(y_{i}^{n}-y^{*}\right)\right)\left(\left(2-\eta_{2}\right)\left(u_{2 i}^{n}-u_{2}^{*}\right)+e_{2}\left(y_{i}^{n}-y^{*}\right)\right) \\
= & -\frac{d_{2} a_{12} \eta_{2}\left(2-\eta_{2}\right)}{2\left(1-\eta_{2}\right) e_{2} a_{21}} \sum_{i=1}^{m}\left(u_{2 i}^{n}-u_{2}^{*}\right)^{2} \\
& +\frac{d_{2} a_{12} e_{2}}{2\left(1-\eta_{2}\right) a_{21}} \sum_{i=1}^{m}\left(y_{i}^{n}-y^{*}\right)^{2}+\frac{a_{12} d_{2}}{a_{21}} \\
& \cdot\left(y_{i}^{n}-y^{*}\right)\left(u_{2 i}^{n}-u_{2}^{*}\right) .
\end{aligned}
$$

Let

$$
V^{n}=V_{1}^{n}+V_{2}^{n}+V_{3}^{n}+V_{4}^{n}
$$

Then,

$$
\begin{aligned}
\Delta V^{n}= & V^{n+1}-V^{n} \\
\leq & \left(-1+\frac{d_{1} e_{1}}{2\left(1-\eta_{1}\right)}\right) \sum_{i=1}^{m}\left(x_{i}^{n}-x^{*}\right)^{2} \\
& +\left(-\frac{a_{12}}{a_{21}}+\frac{d_{2} a_{12} e_{2}}{2\left(1-\eta_{2}\right) a_{21}}\right) \sum_{i=1}^{m}\left(y_{i}^{n}-y^{*}\right)^{2} \\
& +\frac{d_{1} \eta_{1}\left(\eta_{1}-2\right)}{2\left(1-\eta_{1}\right) e_{1}} \sum_{i=1}^{m}\left(u_{1 i}^{n}-u_{1}^{*}\right)^{2} \\
& +\frac{d_{2} a_{12} \eta_{2}\left(\eta_{2}-2\right)}{2\left(1-\eta_{2}\right) e_{2} a_{21}} \sum_{i=1}^{m}\left(u_{2 i}^{n}-u_{2}^{*}\right)^{2} \\
& -D_{1} \sum_{i=1}^{m-1}\left(\sqrt{\frac{x_{i+1}^{n}}{x_{i}^{n}}}-\sqrt{\frac{x_{i-1}^{n}}{x_{i}^{n}}}\right)^{2} \\
& -D_{1}\left(\sqrt{\frac{x_{m}^{n}}{x_{1}^{n}}}-\sqrt{\frac{x_{1}^{n}}{x_{m}^{n}}}\right)^{2}+o(1)+o\left(\rho_{1}\right) \\
& -D_{2} \frac{a_{12}}{a_{21}} \sum_{i=1}^{m-1}\left(\sqrt{\frac{y_{i+1}^{n}}{y_{i}^{n}}}-\sqrt{\frac{y_{i-1}^{n}}{y_{i}^{n}}}\right)^{2} \\
& -D_{2} \frac{a_{12}}{a_{21}}\left(\sqrt{\frac{y_{m}^{n}}{y_{1}^{n}}}-\sqrt{\frac{y_{1}^{n}}{y_{m}^{n}}}\right)^{2}+o(1)+o\left(\rho_{2}\right) .
\end{aligned}
$$

If $\left(d_{1} e_{1} / 2\left(1-\eta_{1}\right)\right) \leq 1,\left(d_{2} e_{2} / 2\left(1-\eta_{2}\right)\right) \leq 1,\left(d_{1} \eta_{1}\left(\eta_{1}-\right.\right.$ 2)/2(1- $\left.\left.\eta_{1}\right) e_{1}\right) \leq 0$ and $\left(d_{2} a_{12} \eta_{2}\left(\eta_{2}-2\right) / 2\left(1-\eta_{2}\right) e_{2} a_{21}\right) \leq 0$ or $\left(d_{1} e_{1} / 2\left(1-\eta_{1}\right)\right) \leq 1,\left(d_{2} e_{2} / 2\left(1-\eta_{2}\right)\right) \leq 1, \eta_{1}<1$ and $\eta_{2}<1$ hold, $\Delta V^{n} \leq 0$. The proof is completed.

\section{Example and Numerical Simulations}

In the following example, we will show the feasibility of our main results and discuss the effects of feedback controls. Take $i=2$ in the system; we obtain a model with feedback controls as follows:

$$
\left\{\begin{array}{l}
x_{i}^{n+1}=x_{i}^{n} \exp \left(r_{1}-x_{i}^{n}-a_{12} y_{i}^{n}-d_{1} u_{1 i}^{n}\right)+D_{1} \nabla^{2} x_{i}^{n}, \\
y_{i}^{n+1}=y_{i}^{n} \exp \left(r_{2}+a_{21} x_{i}^{n}-y_{i}^{n}-d_{2} u_{2 i}^{n}\right)+D_{2} \nabla^{2} y_{i}^{n}, \\
u_{1 i}^{n+1}=\left(1-\eta_{1}\right) u_{1 i}^{n}+e_{1} x_{i}^{n}, \\
u_{2 i}^{n+1}=\left(1-\eta_{2}\right) u_{2 i}^{n}+e_{2} y_{i}^{n}, \\
i=1,2
\end{array}\right.
$$

with the periodic boundary conditions 


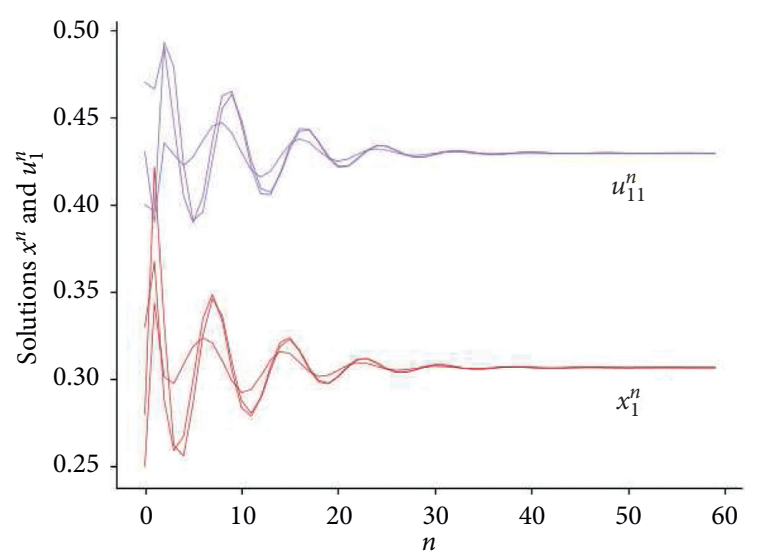

(a)

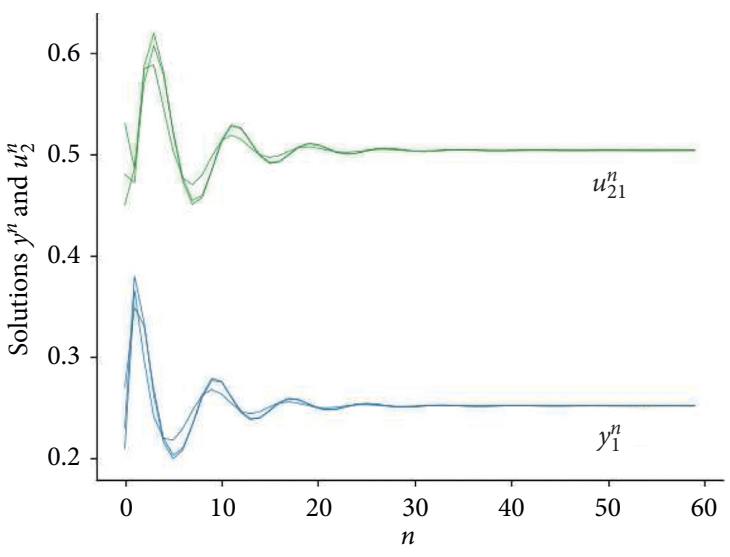

(c)

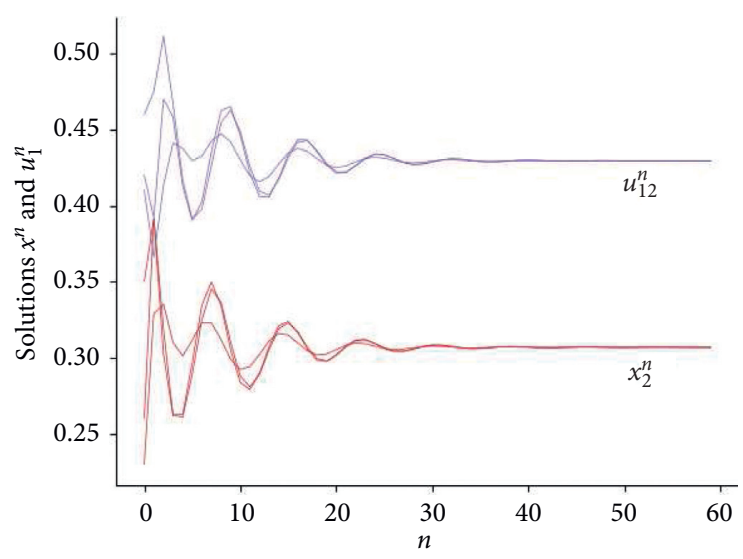

(b)

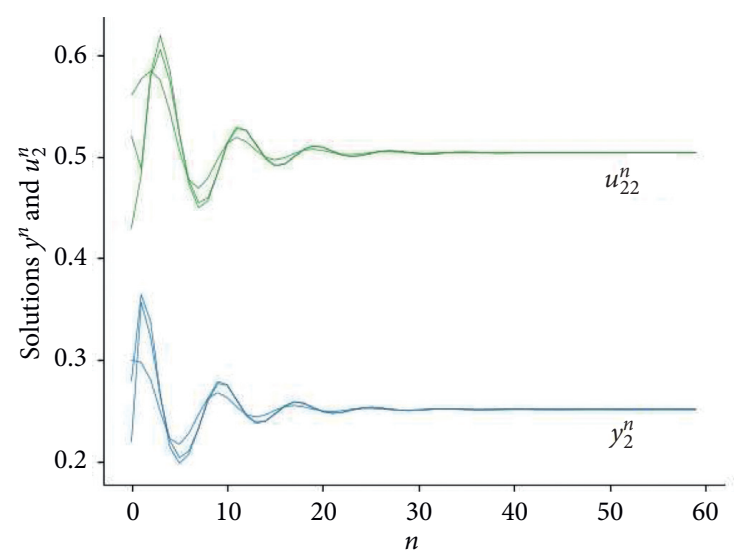

(d)

FIgURE 1: Dynamic behaviors of systems (36) and (37) with three sets of different initial conditions when $r_{1}=1.2$, $r_{2}=1, a_{12}=0.8, a_{21}=0.3, e_{1}=0.7, e_{2}=0.6, \eta_{1}=0.5, \eta_{2}=0.6, d_{1}=1, d_{2}=1, D_{1}=0.3$, and $D_{2}=0.4$.

TABLE 1: Different initial values for $x_{1}^{n}, x_{2}^{n}, y_{1}^{n}, y_{2}^{n}, u_{11}^{n}, u_{12}^{n}, u_{21}^{n}, u_{22}^{n}$.

\begin{tabular}{ccccccccc}
\hline & $x_{1}^{0}$ & $x_{2}^{0}$ & $y_{1}^{0}$ & $y_{2}^{0}$ & $u_{11}^{0}$ & $u_{12}^{0}$ & $u_{21}^{0}$ & $u_{22}^{0}$ \\
\hline 1 & 0.22 & 0.35 & 0.27 & 0.22 & 0.40 & 0.46 & 0.45 & 0.52 \\
2 & 0.33 & 0.26 & 0.23 & 0.28 & 0.47 & 0.42 & 0.48 & 0.43 \\
3 & 0.25 & 0.23 & 0.21 & 0.30 & 0.43 & 0.41 & 0.53 & 0.56 \\
\hline
\end{tabular}

$$
\left\{\begin{array}{l}
x_{0}^{n}=x_{2}^{n}, \\
x_{1}^{n}=x_{3}^{n}, \\
y_{0}^{n}=y_{2}^{n}, \\
y_{1}^{n}=y_{3}^{n} .
\end{array}\right.
$$

To illustrate our purposes, the parameter values are chosen as follows (the choice of parameter values is hypothetical with appropriate units and not based on data): $r_{1}=$ $1.2, r_{2}=1, a_{12}=0.8, a_{21}=0.3, d_{1}=1, d_{2}=1, D_{1}=0.3, D_{2}=0.4$, $e_{1}=0.7, e_{2}=0.6, \eta_{1}=0.5$, and $\eta_{2}=0.6$; then, there is only a unique positive equilibrium $E^{*}\left(x_{1}^{*}, x_{2}^{*}, y_{1}^{*}, y_{2}^{*}, u_{11}^{*}, u_{12}^{*}, u_{21}^{*}\right.$, $\left.u_{22}^{*}\right)=(0.3181,0.3181,0.5487,0.5487,0.4453,0.4453,0.5487$, $0.5487)$. It is easy to see that the conditions in Theorem 2 are verified. Dynamic behaviors of systems (36) and (37) with the initial conditions are shown in Figure 1, and three sets of different initial conditions are listed in Table 1. The simulations

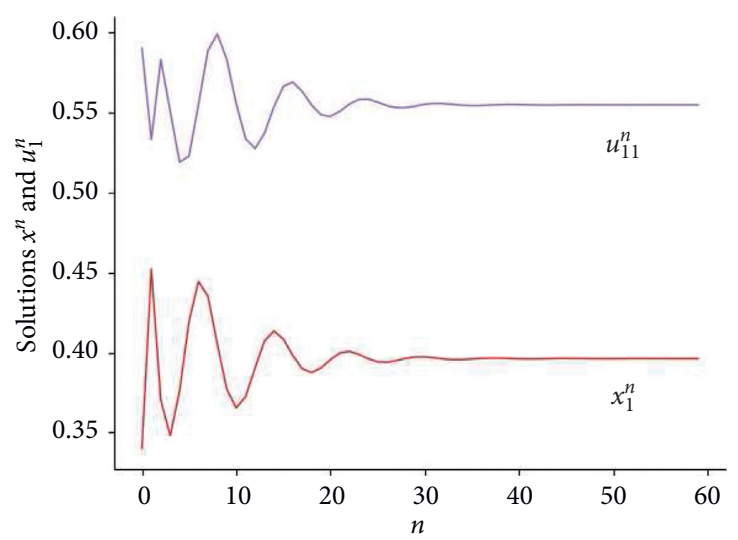

Figure 2: Dynamic behaviors of $x_{1}^{n}$ and $u_{11}^{n}$ for systems (36) and (37) with initial conditions $(0.36,0.34,0.36,0.34,0.28,0.31$, $0.28,0.31,0.59,0.52,0.45,0.54)$ when $d_{2}=0.8$.

can illustrate the fact that the positive equilibrium is globally asymptotically stable.

To explore clearly the dynamical behavior of systems (36) and (37), we investigate the effect of diffusion parameter $d_{2}$ by keeping other parameters of the system fixed. Figure 2 exhibits in detail an interesting situation when 


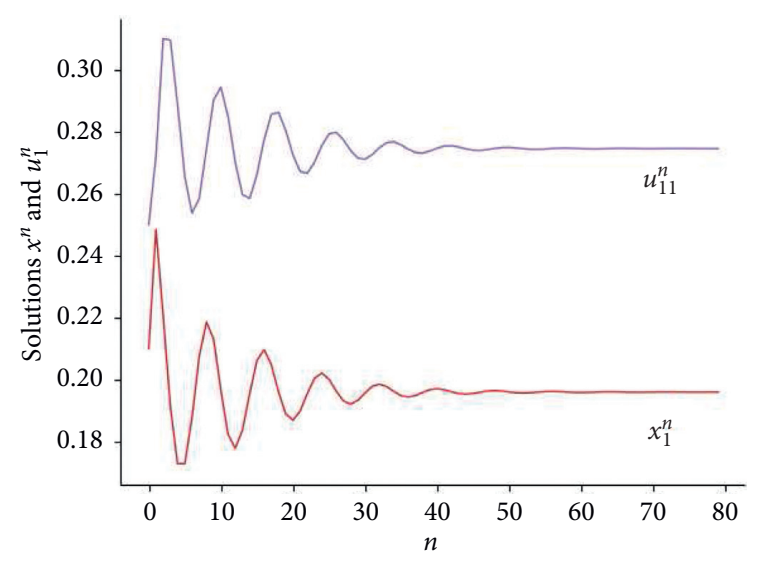

Figure 3: Dynamic behaviors of $x_{1}^{n}$ and $u_{11}^{n}$ for systems (36) and (37) with initial conditions $(0.16,0.21,0.16,0.21,0.21,0.26,0.21$ $, 0.26,0.25,0.22,0.45,0.50)$ when $d_{2}=1.2$.

$d_{2}=0.8\left(r_{1}=1.2, r_{2}=1, a_{12}=0.8, a_{21}=0.3, d_{1}=1, D_{1}=\right.$ $0.3, D_{2}=0.4, e_{1}=0.7, e_{2}=0.6, \eta_{1}=0.5, \eta_{2}=0.6$ ), in which the solutions converge faster than Figure 1 . With the increase of $d_{2}$, the solutions converge more slowly, as depicted in Figure 3. For the sake of convenience, only the dynamical behavior of $x_{1}^{n}$ and $u_{11}^{n}$ with a group of initial values is shown in Figures 2 and 3. The simulation results of adjusting feedback control coefficients $d_{1}, e_{1}$, and $e_{2}$ are omitted.

\section{Conclusions and Discussion}

This paper investigates the global asymptomatic stability of the unique positive equilibrium of a discrete diffusion model with the periodic boundary conditions and feedback control. The condition to ensure the nonnegativity and boundedness of the solutions of the discrete model is discussed, and the globally asymptotical stability of the positive equilibrium is proved. Through comparing numerical simulations, we notice that when we improve the feedback control coefficients, the solutions will converge more slowly. It follows that we can adjust the rate of convergence by choosing suitable values of feedback control variables. Such work may also be applied to other discrete diffusion models.

It should be noted that there is only a basic condition obtained to guarantee the existence of positive solution. Judgement sentence $x_{i}^{n}, y_{i}^{n}, u_{1 i}^{n}, u_{2 i}^{n} \geq 0$ should be added into the simulation programs under the conditions of $e^{r_{j}}-2 D_{j} \geq 0$ and $\eta_{j} \leq 1, j=1,2$. Further improvements are needed.

In this study, all of the coefficients of the model system are constant; in many situations, they can be assumed to be nonconstant bounded nonnegative sequences such as periodic positive sequences which can reflect the seasonal fluctuations [19]. On the other hand, time delays can have a great influence on species populations. It is worthy to consider the nonautonomous space-time discrete Lotka-Volterra system with feedback control.

Different control schemes, such as switching control, constraint control, and sliding control, can be applied to the system models. Many interesting results can be obtained.
Based on the backstepping recursive technique, a neural network-based finite-time control strategy is proposed for a class of non-strict-feedback nonlinear systems [31], and an event-triggered robust fuzzy adaptive prescribed performance finite-time control strategy is proposed for a class of strict-feedback nonlinear systems with external disturbances [32]. How to apply these control schemes on the discrete diffusion models may be worth considering.

It is well known that noise disturbance is unavoidable in real systems, and it has an important effect on the stability of systems. Also, noise can be used to stabilize a given unstable system or to make a system even more stable when the system is already stable which reveals that the stochastic feedback control can stabilize and destabilize the deterministic systems $[33,34]$. Therefore, it will be interesting and challenging to investigate stabilization or destabilization of nonlinear discrete space-time systems by stochastic feedback control in our future work.

\section{Data Availability}

No data were used to support this study.

\section{Conflicts of Interest}

The authors declare that they have no conflicts of interest.

\section{Acknowledgments}

This research was supported by the Applied Study Program (grant nos. 171006901B, 60204, and WH18012).

\section{References}

[1] J. Xu and Z. Teng, "Permanence for a nonautonomous discrete single-species system with delays and feedback control," Applied Mathematics Letters, vol. 23, no. 9, pp. 949-954, 2010.

[2] O. S. Board, An Ecosystem Services Approach to Assessing the Impacts of the Deepwater Horizon Oil Spill in the Gulf of Mexico, National Academies Press, Washington, DC, USA, 2013.

[3] V. Tiwari, J. P. Tripathi, R. K. Upadhyay, Y.-P. Wu, J.-S. Wang, and G.-Q. Sun, "Predator-prey interaction system with mutually interfering predator: role of feedback control," Applied Mathematical Modelling, vol. 87, pp. 222-244, 2020.

[4] X. Li, X. Yang, and T. Huang, "Persistence of delayed cooperative models: impulsive control method," Applied Mathematics and Computation, vol. 342, pp. 130-146, 2019.

[5] T. Luo, "Stabilization of multi-group models with multiple dispersal and stochastic perturbation via feedback control based on discrete-time state observations," Applied Mathematics and Computation, vol. 354, pp. 396-410, 2019.

[6] W. Qin, X. Tan, M. Tosato, and X. Liu, "Threshold control strategy for a non-smooth Filippov ecosystem with group defense," Applied Mathematics and Computation, vol. 362, Article ID 124532, 2019.

[7] J. Xu, Z. Teng, and H. Jiang, "Permanence and global attractivity for discrete nonautonomous two-species lotkavolterra competitive system with delays and feedback controls," Periodica Mathematica Hungarica, vol. 63, no. 1, pp. 19-45, 2011. 
[8] C. Walter, "Stability of controlled biological systems," Journal of Theoretical Biology, vol. 23, no. 1, pp. 23-38, 1969.

[9] P. Wang, Z. Zhao, and W. Li, "Global stability analysis for discrete-time coupled systems with both time delay and multiple dispersal and its application," Neurocomputing, vol. 244, pp. 42-52, 2017.

[10] Y. Kuang, "Global stability in delay differential systems without dominating instantaneous negative feedbacks," Journal of Differential Equations, vol. 119, no. 2, pp. 503-532, 1995.

[11] Y. Yan and E.-O. N. Ekaka-a, "Stabilizing a mathematical model of population system," Journal of the Franklin Institute, vol. 348 , no. 10 , pp. $2744-2758,2011$.

[12] Y. Muroya, "Global stability of a delayed nonlinear lotkavolterra system with feedback controls and patch structure," Applied Mathematics and Computation, vol. 239, pp. 60-73, 2014.

[13] J. L. Liu and W. C. Zhao, "Dynamic analysis of stochastic lotka-volterra predator-prey model with discrete delays and feedback control," Complexity, vol. 2019, p. 15, Article ID 4873290, 2019.

[14] Q. Zhu and H. Wang, "Output feedback stabilization of stochastic feedforward systems with unknown control coefficients and unknown output function," Automatica, vol. 87, pp. 166-175, 2018.

[15] Q. Zhu, "Stabilization of stochastic nonlinear delay systems with exogenous disturbances and the event-triggered feedback control," IEEE Transactions on Automatic Control, vol. 64, no. 9, pp. 3764-3771, 2019.

[16] K. Kiss and É. Gyurkovics, "LMI approach to global stability analysis of stochastic delayed lotka-volterra models," Applied Mathematics Letters, vol. 104, pp. 106227: 1-6, 2020.

[17] X. Chen and C. Fengde, "Stable periodic solution of a discrete periodic lotka-volterra competition system with a feedback control," Applied Mathematics and Computation, vol. 181, no. 2, pp. 1446-1454, 2006.

[18] C. Niu and X. Chen, "Almost periodic sequence solutions of a discrete lotka-volterra competitive system with feedback control," Nonlinear Analysis: Real World Applications, vol. 10, no. 5, pp. 3152-3161, 2009.

[19] X. Liao, S. Zhou, and Y. Chen, "Permanence and global stability in a discrete $n$-species competition system with feedback controls," Nonlinear Analysis: Real World Applications, vol. 9, no. 4, pp. 1661-1671, 2008.

[20] A. M. Turing, "The chemical basis of morphogenesis," PhiloSophical Transactions of the Royal Society B: Biological Sciences, vol. 237, no. 641, pp. 37-72, 1952.

[21] K. Gopalsamy and P. X. Weng, "Feedback regulation of logistic growth," International Journal of Mathematics and Mathematical Sciences, vol. 16, no. 1, pp. 177-192, 1993.

[22] L. Xu, S. S. Lou, P. Q. Xu, and G. Zhang, "Feedback control and parameter invasion for a discrete competitive lotka-volterra system," Discrete Dynamics in Nature and Society, vol. 2018, p. 8, Article ID 7473208, 2018.

[23] X. W. Li and H. J. Gao, "Robust finite frequency $H_{\infty}$ filtering for uncertain 2-D roesser systems," Automatica, vol. 48, pp. 1163-1170, 2012.

[24] X. W. Li, H. J. Gao, and C. H. Wang, "Generalized kalman-yakubovich-popov lemma for 2-D FM LSS model," IEEE Transaction on Automatic Control, vol. 57, no. 12, pp. 3090-3193, 2012.

[25] J. Zhou, Y. Yang, and T. Zhang, "Global dynamics of a reaction-diffusion waterborne pathogen model with general incidence rate," Journal of Mathematical Analysis and Applications, vol. 466, no. 1, pp. 835-859, 2018.

[26] Y. Yang and J. Zhou, "Global stability of a discrete virus dynamics model with diffusion and general infection function," International Journal of Computer Mathematics, vol. 96, no. 9, pp. 1752-1762, 2019.

[27] P. Liu and S. N. Elaydi, "Discrete competitive and cooperative models of lotka-volterra type," Journal of Computational Analysis and Applications, vol. 3, no. 1, pp. 53-73, 2001.

[28] L. L. Meng and Y. T. Han, "Bifurcation, chaos, and pattern formation for the discrete predator-prey reaction-diffusion model," Discrete Dynamics in Nature and Society, vol. 2019, p. 9, Article ID 9592878, 2019.

[29] R. M. May and G. F. Oster, "Bifurcations and dynamic complexity in simple ecological models," The American Naturalist, vol. 110, no. 974, pp. 573-599, 1976.

[30] Z. Zhou and X. Zou, "Stable periodic solutions in a discrete periodic logistic equation," Applied Mathematics Letters, vol. 16, no. 2, pp. 165-171, 2003.

[31] K. K. Sun, J. B. Qiu, H. R. Karimi, and H. J. Gao, "A novel finite-time control for nonstrict feedback saturated nonlinear systems with tracking error constraint," IEEE Transactions on Systems, Man, and Cybernetics: Systems, pp. 1-12, 2020.

[32] K. K. Sun, J. B. Qiu, H. R. Karimi, and Y. L. Fu, "Eventtriggered robust fuzzy adaptive finite-time control of nonlinear systems with prescribed performance," IEEE Transactions on Fuzzy Systems, 2020.

[33] X. Mao, "Almost sure exponential stabilization by discretetime stochastic feedback control," IEEE Transactions on Automatic Control, vol. 61, no. 6, pp. 1619-1624, 2016.

[34] Q. Zhu and T. Huang, "Stability analysis for a class of stochastic delay nonlinear systems driven by G-Brownian motion," Systems \& Control Letters, vol. 140, Article ID 104699, 9 pages, 2020. 